\title{
Theory Before Definition in Martial Arts Studies
}

\section{Dealing with Disciplinary Difference}

The matter of value that was broached in the previous chapter demands further interrogation. To do so here, let me begin with an anecdote. I was once invited to contribute a chapter to a collection being prepared on martial arts and embodied knowledge. When all the draft chapters were in and the editors were happy with the collection, the entire manuscript was then sent off to be assessed by two academic reviewers. Of my own contribution, one reviewer said that the chapter by Bowman was terrible, not publishable, and should be rejected. The other reviewer said that the chapter by Bowman was the best contribution to the volume, and greatly enhanced and enriched it. Faced with two diametrically opposed views from two presumably equally reliable peer reviewers, ${ }^{1}$ the editors themselves held the casting vote. They decided that they liked the chapter overall, thought it had value, and wanted to include it. But they elected to share the reviews with me and invited me to make any changes I thought appropriate in light of them.

The experience of receiving such polarised views was educational. I share this anecdote here to introduce a cluster of interrelated issues. These start

${ }^{1}$ At the time, the emerging field that we now call martial arts studies was yet to be established, and the editors later commented that they had actually struggled to find suitable academics to act as peer reviewers who were not already contributors to the collection itself. Today, there would be peer reviewers aplenty for such a collection. This could be taken to demonstrate many things, including the proposition that the establishment of an academic field involves not only the establishment of (new) shared objects of attention, shared problematics and shared methodologies, but also the production of (new) academic subjects - i.e., individual scholars with a recognisable disciplinary identity, conferred or established reciprocally in the process of emergence of the discourse itself.

How to cite this book chapter:

Bowman, P. 2019. Deconstructing Martial Arts. Pp. 33-59. Cardiff: Cardiff University Press. DOI: https://doi.org/10.18573/book1.c. License: CC-BY-NC-ND 4.0 
with the matter of how to establish value in an emergent academic discourse, the problematic of bias attendant to all acts and processes of evaluation and verification, and the fact that the shape, form, borderlines, organisation and orientation of academic fields are neither natural nor inevitable. Rather, these emerge in negotiation with decisions made by a host of agents and agencies, including academics, editors, reviewers, research councils, funding bodies, and publishers, all of whom make their evaluations with reference to established criteria and values. Accordingly, decisions as to what good or bad work looks like, and what 'deserves' to be published, are themselves reflective of values tied into interpretations of what good, correct or proper academic work in this field 'should' look like.

This does not mean that everything is already decided or overdetermined by pre-established 'structures' or 'systems'. Rather, it means that senses of propriety, validity, appropriateness, fit, and so on, are always establishments or achievements that are ongoing, in negotiation, subject to dispute, up for question, challenge, revision and review. Such negotiation and renegotiation can be perceived in all academic disciplines, but it is inevitably more cacophonic in newly emerging fields, where senses of tradition and tacit agreements about convention have yet to be set.

This is the situation of martial arts studies today, in which huge disciplinary differences are palpable from one work to the next. Such vast differences are present because even though the emergence of the field is being driven by a sense of shared and communal investment in an object ('martial arts'), this shared interest is not yet matched by anything like a shared approach. In other words, the shared academic interest in 'martial arts' is currently drawing together academics from many very different fields. Yet the deceptiveness of the term 'martial arts' combined with the diversity of this community, with its myriad premises, multiple perspectives, methods and orientations, seems to necessitate the creation of some kind of consensus around the object, field and approach to 'martial arts'. Hence, understandably, people feel the need to establish a definition of martial arts (Jones 2002; Lorge 2012, 2016; but see also Judkins 2016b).

Because of this perceived necessity, at this point, many works would move directly into a discussion of definitions, attempting to settle the matter of which definition of martial arts should and should not be used, and where and when (Channon 2016; Lorge 2016). However, rather than entering into the discussion about how best to define martial arts, in what follows I will instead argue that the question of the definition of martial arts is both a distraction and a red herring for the emerging field of martial arts studies. The more pressing task, I argue, is not the establishment of a consensus around the definition of our object. Rather, it is the establishment of a shared, circumspect, literate, analytical and theoretically informed critical discourse with rigorously formulated problematics that can contribute in diverse ways to both academic and public debates. 
In short, I argue (somewhat against the current of recent debates, and even against the grain of many academic approaches) that we do not need to define martial arts at all. Rather, I propose that we need to theorise the entire field or nexus of research, including the place, point and purpose of definition within it. Indeed, my contention is that if we allow ourselves to be animated by defining martial arts without both theorising and constructing the field, then martial arts studies may founder and fail, like so many past attempts to establish an academic field of study for martial arts. ${ }^{2}$

\section{Approaching Martial Arts Studies}

Before following through on this, some further reflection on my opening anecdote seems called for. Ironically, even back at the time of this brush between my work and two border guards at the gates of a then unclear and embryonic field, I had already long accepted Roland Barthes' argument that readers can and will have very different responses to the same text (Barthes 1977). But I had never encountered such symmetrically opposed opinions from scholars I had presumed must work to some degree in the 'same' area, or at least close enough i.e., holding an academic interest in 'martial arts' refracted through one or another approach of the arts or humanities. Even though I already believed I knew that academic disciplines are spaces of argumentation and disagreement rather than consensus, the vastly differing verdicts nonetheless surprised me. Today, I am no longer surprised by the appearance of such difference in what is still a very young and uncertain field. Indeed, as mentioned, encountering extreme disciplinary differences is currently our daily bread. The question is: Is such diversity simply something to be either shrugged off or celebrated, or might it harbour a problem? What might it mean if the object and field of martial arts studies continues to be conceived of very differently by different people from different disciplinary backgrounds? In short, is it a problem that we are still frequently experiencing such widely differing approaches to the academic study martial arts?

On the one hand, no. There will always be disciplinary difference, and even vast differences in conceptuality and orientation within 'the same' discipline or field. Different academic origins and kinds of training bring with them differing questions, differing objects of attention, differing values, methods, and so on. And, for the foreseeable future, martial arts studies will inevitably be built

\footnotetext{
${ }^{2}$ For an important and valuable contrast to this argument, see Peter Lorge's recent work (Lorge 2016), in which he argues against using theory - because it is difficult and off-putting - and instead for the virtues of deepening and refining historical knowledge. As Lorge sees it, deeper historical knowledge can both enrich martial artists' practice and clearly illustrate to the academic community the value of martial arts as a valid topic of academic study. I believe it is possible to concede Lorge's points and still argue for the value and necessity of theory 'before' or 'beneath' this.
} 
from work and approaches hailing from different disciplines. Moreover, no one will ever be surprised, for instance, if a study of martial arts as they appear in one or more works of literature differs significantly from a study focused on questions of experiments in or around pedagogy (compare Liu 2011 with Lefebvre 2016 for instance). So where might the problem come in?

Far be it from me to advocate any kind of unitary, univocal, mono-disciplinary or monoculturalist approach in martial arts studies. That would be neither desirable nor possible. Nonetheless, in order for a field or discipline to emerge and survive, there must be coherent and meaningful internal (community) and external (cross-disciplinary) discourses and exchanges. In order for this to happen, the matter of what we might call 'the approach' is important. There are stakes and consequences attached to the matter of the paradigms that organise our efforts.

To illustrate, one might briefly consider the possible reasons for the repeated failure of attempts to create a field of academic study of and for martial arts. Most famously, of course, Richard Burton in the $19^{\text {th }}$ Century and Donn Draeger later in the $20^{\text {th }}$ Century attempted to found and ground an academic discipline that they called 'hoplology' (for an overview, see Spencer 2011). However, this or these projects repeatedly foundered. The question is: Why?

Even more pertinently, perhaps, is the related question of why a connected field of martial arts studies took until today to begin to emerge at all. Consider the fact that over the last two decades it became increasingly easy to carry out online academic searches and to discover that all sorts of different kinds of studies of all sorts of issues involving martial arts are being carried out across a surprising number of different disciplines. Yet there have been few sustained dialogues and fewer dedicated spaces for the academic study of martial arts. ${ }^{3}$

My contention is that the matter of the approach or paradigm is central to both questions. It relates not only to all failed past attempts to establish any kind of martial arts studies but also to the stubborn non-appearance of martial arts studies until today, despite scattered studies of martial arts in diverse disciplines.

To consider the recent situation first: There is a sense in which the very heterogeneity of the ways of approaching martial arts - the very richness of the potential field - may paradoxically have played a part in preventing the creation of a single interconnected, interacting field. The logic of this proposition is as follows: The creation of an academic discourse requires the emergence of shared problematics and discussions around - at the very least - matters of which questions are to be asked and which methodologies are best suited for their exploration. Yet, in recent decades, although there have been a great number of academic studies on all manner of things to do with martial arts, no

\footnotetext{
${ }^{3}$ The long-running Electronic Journals of Martial Arts and Sciences is a noteworthy project that has attempted to construct such dialogues and spaces (http://ejmas.com/). I defer a discussion of this project here, however, in order to focus on more 'stark' examples for clarity.
} 
single field or conversation has emerged, because of the very heterogeneity of approaches to radically heterogeneous questions. (Moreover, far from informing, enlivening and expanding academic discourses on martial arts, the heterogeneity of approaches and diversity of kinds of work actually seems to have prevented many people from reading, engaging, or even being aware of the plethora of academic literature being produced on martial arts across the disciplines. Works continue to appear that present themselves as if they are the first to deal with the martial arts. Whether proceeding by making grand proclamations to this effect or by lacking a basic literature review, the net result is the same.)

What seems key to disciplinary emergence is a sense of a shared project. But this does not mean that a field demands a unitary or univocal approach. Far from it. Taking too limited a conception of the object and of the field, particularly when this is combined with too limited or problematic an approach, can equally stymie growth. This might be illustrated by a consideration of perhaps the most well-known past attempt to establish a field for the academic study of martial arts - hoplology.

\section{Hoplological Hopes}

Hoplology is surely the most famous example of the failure of martial arts studies to attain a stable and sustainable academic presence. According to the website of the International Hoplology Society, hoplology was founded by Sir Richard F. Burton in the $19^{\text {th }}$ Century. However, it then (says the website) 'remained dormant' until Donn Draeger picked up the baton at some unspecified point after the 1960s, a baton he carried until his death ('About the International Hopology Society' n.d.). The International Hoplology Society is now based in Hawaii and presents itself as 'an independent, not-for-profit organization' which 'offers its services to scholars, universities, museums, collectors, private and governmental organizations, writers and publishers around the world' ('About the International Hopology Society' n.d.).

Given this evidence of its continued and current existence, readers may be surprised by my claim that hoplology is a failed academic project. Hoplology still exists. The published work of Donn Draeger itself is of mythic status in most narratives of the history of Western attempts to establish serious and reliable scholarly knowledge of East Asian martial arts. Nonetheless, what provides the clearest evidence that the project failed is the lack of any significant academic presence for hoplology. It is neither a discipline, nor a discourse, nor an unfolding research programme, nor an interdisciplinary nexus of debate. The fact that hoplology continues to haunt us in the form of the hopes and aspirations of its proponents does not change the fact that as a field of study it never really made it to where any such field of study most wants to be - the university. The university was always where Draeger and other proponents wanted hoplology to be. But it never really made it. 
It 'never really made it' for lots of different kinds of reasons. There were of course both personal and 'political' elements at work that arguably hampered Draeger's attempts to get hoplology into a university (Miracle 2015). But my contention is that, more significantly, there have always been fundamental obstacles to its academic survival, and that these have always boiled down to its flawed conceptions of its object and its flawed theoretical orientations - in other words, its flawed paradigm and approach.

There are many possible ways to illustrate the conceptual and orientation problems at the heart of hoplology. For the sake of brevity and clarity, I will limit myself to one quick example. This is taken from the front-page text of the International Hoplology Society website, ${ }^{4}$ which proudly trumpets the 'three axioms of hoplology'. These three axioms are:

1. The foundation of human combative behavior is rooted in our evolution. To gain a realistic understanding of human combative behavior, it is necessary to have a basic grasp of its evolutionary background.

2. The two basic forms of human combative behavior are predatory and affective. Predatory combative behavior is that combative/aggressive behavior rooted in our evolution as a hunting mammal. Affective combative behavior is that aggressive/combative behavior rooted in our evolution as a group-social animal.

3. The evolution of human combative behavior and performance is integral with the use of weapons. That is, behavior and performance is intrinsically linked to and reflects the use of weapons. ('About the International Hopology Society' n.d.)

From any academic point of view, the fundamental problem with these axioms is that they are not academic. Rather, they are tenets, beliefs, and assertions. They may appear scientific on first glance, but they are actually scientistic. Specifically, they reflect an attempt to align hoplology with the controversial (and equally dubious) field of sociobiology (Wilson 1975), which itself has long been accused, among other things, of scientism and biological determinism (Schreier et al. n.d.; Bethell n.d.). ${ }^{5}$ The function of these scientific-sounding

${ }^{4}$ Although focusing on such an example may be open to the criticism that it has not been taken from a properly academic context and so should not be subjected to academic critique, nonetheless this example has been selected because these are words that have been placed 'front and centre' and presented as expressing the heart of the hoplological academic project.

${ }^{5}$ The semi-autobiographical pseudo-academic book The Professor in the Cage: Why Men Fight and Why We Like To Watch by Jonathan Gottschall is perhaps the most well-known recent iteration of this kind of deeply problematic approach (Gottschall 2015). The book, appropriately, starts from the failure of an English professor's ongoing project to persuade anyone to use ideas from evolutionary biology in literary studies. From this failure, Gottschall turns to his stagnating academic career and the birth of his interest in MMA. In all of this, the book applies simplistic sociobiologistic ideas to the subject of 'fighting'. There is much that might be said about the limitations and skewing effects of all such pseudo-, crypto- and actual sociobiologistic approaches, and they warrant sustained critique. But such critiques should 
'axioms' on the homepage is to gesture to the society's declared commitment to scholarship and research. Unfortunately, this gesture actually demonstrates the opposite: It reveals its constitutively doctrinaire orientation. As such, the text commits quite a few academic crimes, which all effectively add up to a kind of unintentional (but certain) self-ostracising and auto-abdication from the world of serious academic debate and discussion.

Of course, neither Burton nor Draeger authored these words. But it is clear that the table was set and the door opened to welcome them in advance by the kinds of approach common to hoplology since the beginning. The problem is that this is such a limited raison d'etre, articulated with reference to and in terms of a very limited and problematic deployment of an already problematic set of contentions, that it will always be highly unlikely to pass as academic in any field or context. Few, if any, $20^{\text {th }}$ or $21^{\text {st }}$ Century academic journals, for instance, would accept any allegedly academic article that proceeded according to such 'axioms' (as illustrated by the aforementioned case of Gottschall [2015]).

Rather than this, in order to thrive within academia, what is required is something very different. Specifically, martial arts studies must emerge as a coherent communicative and self-sustaining field of meaningful and productive exchanges and interactions that might be diversely relevant. To achieve this, it will be necessary to undertake a sustained and explicit examination of, and engagement with, the stakes and consequences of the different conceptualisations, orientations and methods available to the field. This implies a sustained reflection on premises, remits, orientations and methods, along with ongoing dialogues with other disciplines and the principled awareness of other established and unfolding approaches across academia. Any conceptualisation of the field that starts out as an apologetic exercise for only a single set of assumptions or methods by definition cannot do this and will be highly unlikely to attract wider academic interest.

This chapter now seeks to contribute to such a reflection by moving away from failed projects like hoplology and discussing instead some significant recent contributions to the crucial debate about what martial arts studies is and how it might elaborate itself and develop. Before engaging with these contributions, however, it will be worthwhile to give some more attention to the matter of the significance for academic discourses of differing approaches and values.

\section{Moving from 'Thing Itself' to 'Field Itself'}

One helpful way to understand why differences of opinion and orientation will always occur within academic disciplines and discourses is proposed by Jacques Derrida (Derrida and Ferraris 2003). Derrida proposes that academic

be careful to avoid being dragged into a scientistic cul-de-sac. There are far better approaches to 'fighting' available than those which rush naively and crudely to ideas of evolutionary advantage (see, for examples, Jackson-Jacobs 2013 and Gong 2015). 
fields are essentially always at war with themselves. The reason for this is that they essentially construct both their own objects and approaches and their own yardsticks for evaluating them. In this sense, a discipline essentially 'constructs the object of argumentation and the field of argumentation itself' (Arditi 2008, 115). There is no immutable or incontestable fixed point outside of the discipline's own discourse from which to adjudicate anything that takes place within it. But what takes place within it depends on a host of variables, including preferences in terms of premises, protocols, practices, procedures, and so on. Therefore, Derrida proposes that:

A field is determined as a field of battle because there is no metalanguage, no locus of truth outside the field, no absolute and ahistorical overhang; and this absence of overhang - in other words, the radical historicity of the field - makes the field necessarily subject to multiplicity and heterogeneity. As a result, those who are inscribed in this field are necessarily inscribed in a polemos, even if they have no special taste for war. There is a strategic destiny, destined to stratagem by the question raised over the truth of the field. (Derrida and Ferraris 2003, 13)

Any academic field is 'a field of battle because there is no metalanguage, no locus of truth outside the field'. This 'makes the field necessarily subject to multiplicity and heterogeneity'. Hence, when faced with divergent opinions or evaluations of any given approach, argument, assessment or experiment within a field, there can be no simple appeal to any higher authority outside the field. ${ }^{6}$ After all, how could anyone outside of a field be universally acknowledged as existing or operating in an informed, experienced or expert enough fashion to adjudicate on what takes place within the field? Do scientists ask philosophers to adjudicate on and decide the value of their methods and findings? Do lawyers? Kant thought that all fields could be interrogated and, in a sense, audited by philosophy. But do those working in fields other than philosophy agree? Indeed, do philosophers really (still) feel entitled, informed or expert enough to do so?

Of course, there are many crossovers and connections between certain fields. Work in one discipline often incorporates elements developed in other disciplines. Economics is often heavily involved in the use of mathematics, for instance. And the academic study of visual art regularly calls upon the approaches and insights of such fields as history, philosophy, cultural theory, sociology, and so on. But such crossovers, connections or collaborations are neither entirely free, nor inevitable, nor established without a battle

\footnotetext{
${ }^{6}$ During the 1980s, much was made of the fact that such a perspective reveals that there is always an aporia at work in the legitimation of knowledge (Derrida 1992), a kind of ineradicable tautology, and even what Lyotard called a 'legitimation crisis in knowledge' (Lyotard 1984).
} 
or disagreement. Rather, such connections are contingent achievements, produced either through a sense of 'obviousness' or appeals to norms (as in, 'Of course the study of art and the study of history overlap and interact') or through the effort of making the case for the validity of their connection (as in, 'Perhaps recent advances in meteorology could be applied to help us learn more about cultural dynamics').

Currently, art history rarely appeals to mathematics for justification or corroboration of the knowledge produced in its own disciplinary space. Although it is not impossible or inconceivable, any move to make the discourse or discipline of art history reliant upon mathematics - or subject to any kind of mathematical validation - would be met with considerable resistance within that field. To propose that the academic discourse around fine art, art history, and so on, should be subject to mathematical procedures would provoke a vehement battle. But the point to be made here is that this battle would merely be different in scale or intensity, not in kind, from the ongoing day to day disagreements within the academic study of fine art and art history around such matters as whether, say, the psychoanalytic paradigm developed in the wake of the work of Jacques Lacan is of more use to art scholars than the sociological paradigm of Pierre Bourdieu, and so on.

Again, these are battles around the question of the paradigm or paradigms that structure a field. The choice of paradigm determines the kind of questions that can be asked, the type of work that will seem to 'need' to be done, and the ways in which such work will be approached.

Reflecting on the ways that academic disciplines and universities work, Derrida argued that what takes place within academic discourses involves 'not an opposition between the legitimate and the illegitimate, but rather a very complicated distribution of the demands of legitimacy' (Derrida 2003, 18). At the very least, then, the determination of such matters as best versus worst is no simple matter. It does not easily come down to a clear question of whether something is 'right' or 'wrong. For, if there is no fixed point outside of and transcending the field, then the source of the determination of such values can only come from within the field itself - from among the paradigms constructed within it.

The problem is that because there will always be more than one paradigm in play (and in process), there will be no sustained consensus arising within the field. In a sense, there are only ever shared, modified or replaced problematics, and rarely any widely held consensus about the formulation of the object, the parameters of the problem, or the framework for any exploration or method of approach.

The proposition that academic disciplines are battlegrounds may either disappoint or delight. It may disappoint those who cleave to the idea that academic disciplines principally trade in the establishment of truth about reality, and that they find out and know more and more about truth and reality as time goes on and as the discipline progresses. Conversely, the idea of disciplines as 
battlegrounds may appeal to others, and for any number of reasons. However, it is important to point out that the type of 'war' being formulated by Derrida here is not some Darwinian or neoliberal notion of 'survival of the fittest'. Rather, Derrida is making a claim about the inevitable and inescapable emergence of pluralities of voices, positions and styles of attempting to establish or verify things within disciplines.

This depicts a condition of incessant and interminable disagreement, in which not only are there no absolute or eternal winners, there are not even agreed criteria for determining what notions like 'survival', 'demise' or indeed 'fittest' might possibly mean. (Has hoplology 'survived'? In what way? Is it 'the fittest'? For what?) In this kind of context, there will always be more to any disagreement than one matter or one issue. Indeed, 'disagreement', in this sense, can usefully be formulated as 'less a confrontation between two established positions - as in the case of a debating society - than an engagement between "parties" that do not antedate their confrontation. A disagreement constructs the object of argumentation and the field of argumentation itself' (Arditi 2008, 115).

In academia, the mode and manner of our argumentation, as well as the very object of our attention itself, must be understood to be particular kinds of institutional constructs. Our objects are 'disciplinary objects', essentially invented within, or at least 'worked over' by, our own discourses (Mowitt 1992). Our approaches to them are constructs too. This is so even though many people seem to believe that academic disciplines and fields just happen, that they are born spontaneously or emerge ineluctably in response to external realities of the world. However, this is not at all the case. Academic subjects are not born, they are made. ${ }^{7}$

\section{The Paradigms of Martial Arts Studies}

In light of this, it is important to realise that an early and essential challenge for the nascent field of martial arts studies was always going to be the field itself. That is to say, at the same time as exploring and engaging with problematics within the field, it is also necessary to more clearly and indeed securely establish martial arts studies as a field of study, that is, as a legitimate field of study (Bowman 2015a; Wetzler 2015).

\footnotetext{
${ }^{7}$ There are many accounts of these processes. See, for example, Anderson on the formation of English Literature as a global discipline (Anderson 1991), Hall on the formation of cultural studies (Hall 1992), Fabian on how anthropology constructs its objects (Fabian 1983), Chow on the invention of film studies (Chow 2007), or, perhaps most famously, Foucault on the invention of psychiatry (Foucault 1989). Indeed, as one commentator put it to me: Why should there be a field of martial arts studies, distinct from the wider study of movement, performance and embodied knowledge?
} 
This matter may not seem to amount to too much of a serious problem, given the abundant empirical evidence that martial arts studies is a field that is mushrooming internationally. There are currently conferences and publications appearing in many languages in many countries. But the fact that this is happening without much in the way of a conversation about how to study martial arts is troubling (Bowman 2015a; Wetzler 2015; Judkins 2016a). History is littered with failed attempts to establish any kind of coherent and sustainable academic discourse of martial arts studies. As I have been suggesting, perhaps this is in large part because of a lack of sustained communal effort to forge conceptual development via cross-disciplinary dialogues.

Moreover, in the present moment, we should not forget that until very recently one of the most frequently posed questions in and around these waters was: Will martial arts ever be a valid topic of academic study? If today we are hearing a resounding 'Yes!', there nevertheless remain not only 'strictly academic' but also 'pressingly practical' reasons for posing such why and how questions. Different answers produce differing conceptualisations of the aim, object and field, and entail different approaches. So, we need to ask: What are our aims? Which approaches best serve such aims?

In the terms of Thomas Kuhn's now classic approach to understanding the ways that academic knowledge is produced, established and transformed, the emergent field of martial arts studies would currently be classified as 'preparadigmatic' (Kuhn 1962; Nicholls 2010). This is because there is little to no consensus about its objects, orientations, methodologies or approaches. Some connections, crossovers and collaborations across disciplines are being formed, thanks to newly formed research networks, conferences and increasingly visible publications, but the wider field has long been confined to discrete islands of disparate disciplinary approaches in small enclaves. So, although some scholars are now producing works that engage with the question of the approaches and paradigms of martial arts studies, there remains much that still needs to be done to establish anything like a coherent topos. ${ }^{8}$

There is much to be said about this. But what I principally want to emphasize in what follows - in an argument that runs contrary or transverse to many discussions and impulses in and around the field - is that none of this entails a 'need' to define martial arts.

${ }^{8}$ Hence the importance of the question of the paradigms of martial arts studies. From the outset, we must pluralise the question because it is evident from the range of scholarship and avenues of enquiry currently beginning to be explored across the disciplines that differing conceptualisations of both object and field emerge reciprocally with different approaches and orientations. To establish the paradigms of martial arts studies, one approach would be to map current approaches, analyse their orientations and interrogate their current and potential interconnections, in order to generate an overarching awareness of the field in its multiplicity and heterogeneity. Of course, the question that arises here is that of the map itself: What are the characteristics of the lens through which the cartographer is looking? 


\section{Against Definition}

As mentioned, there is a widespread belief in and around the nascent discourses of martial arts studies that a primary and orientating task must be to define martial arts (Monahan 2007; Cynarski 2008; Lorge 2012, 2016; Channon and Jennings 2014; Cynarski, Sieber, and Szajna 2014). The matter of defining martial arts has also prompted some valuable recent reflections on the many problems and issues that it raises (Wetzler 2015; Judkins 2016b; Channon 2016). However, I want to intervene by arguing that this very belief and orientation harbours problems (Bowman 2015a, 2017b). More precisely, my argument is that it is actually an error to think that forging definitions must be primary, or indeed even necessary, in academic work. Often, the belief in the necessity of definition is already an effect of a tacit acceptance that a certain manner, mode or register of academic discourse must be the proper, best or necessary method. Indeed, it arguably boils down to a belief that the only or best kind of academic work is scientific, and that science starts from definitions.

There are at least two problems with this. One problem lies with any attempt to make studies of human life, culture and society emulate science. In our case, this would take the form of trying to force the study of martial arts to conform to a certain (scientistic) conception of science. For it is important to be aware that scientific approaches are neither the only nor necessarily the best, assuming they are even viable, approaches. (Must we use scientific methods to explore martial arts in/and literature, film, music, gaming, philosophy, religion, gender, identity, or politics, and so on?) The second problem relates to the idea that science starts with definitions. This involves a misunderstanding of science. Science starts from theory. Scientific method always and only boils down to the attempt to test, verify or falsify a theoretical hypothesis. ${ }^{9}$ Such work often seems to involve numbers, but science does not necessarily involve numbers. Some statements about science or elements of it involve numbers. But what is primary in science is theory.

On the other hand, or at the other end of the supposed spectrum of approaches, even putatively non-scientific approaches to any subject also involve theory - whether consciously acknowledged or not, and whether the theory is postulated explicitly (to orientate the work) or whether it emerges out of the work, through different kinds of encounters with 'objects', 'things', 'processes', 'phenomena' or 'stuff' - and regardless of whether we want to call such stuff 'text', 'evidence', 'material', 'archive,' 'fieldwork', 'results' or 'data'. The

\footnotetext{
${ }^{9}$ One reviewer of this chapter challenged my use of the word 'verify' here, as it jars with scientific terminology. However, I have elected to keep the word, because my thinking is more influenced by Jacques Rancière than by scientific method per se. Rancière argues that attempts to establish, prove or argue for something - anything, anywhere - ultimately involve constructing ways of trying to verify (rather than falsify) the proposition, position or belief one is supporting (see for instance Rancière 1992).
} 
belief that such encounters, or any results or statements about any of this, necessarily or properly begins or ends with 'definition' is a misunderstanding. As such, any approach that positions the matter of how to define martial arts as if it is a primary or somehow fundamental question is misconceived or badly formed.

As Alex Channon has recently reminded us (although he argues for the utility of principled moments of definition), definitions quickly produce hierarchies, and help to erect values, borderlines, norms and exclusions (Channon 2016).

\section{For Theory}

Fortunately, early work in the recently established journal Martial Arts Studies has, from the outset, attempted to move beyond the (dis)orientation caused by becoming trapped in the taxonomical labours associated with defining. Issue One of Martial Arts Studies, for instance, contained several different efforts to conceptualise the field and to work out ways that it could profitably and productively develop (Bowman 2015b; Wetzler 2015; D.S. Farrer 2015; Barrowman 2015b). Significant among these is Sixt Wetzler's 'Martial Arts Studies as Kulturwissenschaft: A Possible Theoretical Framework' (Wetzler 2015). ${ }^{10}$ This article is a particularly notable contribution to the field, to which I would now like to turn.

In his article, Wetzler carries out a number of important tasks. He identifies the pitfalls that can arise when academics use the object-, folk-, or practitioner-language of the practices that they are taking as their objects of study. From here, he broaches the problem of adequate academic terminology, asking: What terms should scholars use when talking about this or that aspect of martial arts in/and/as culture, politics, history or society? He then argues that academic terms should surely not be the same as the terms and concepts used by practitioners themselves, either to characterise what they do or to carve up the conceptual spectrum of categories and hierarchies. This discussion moves Wetzler into a reflection on the well-worn problems of conceptualisation and surprise, surprise - definition.

In an important move, however, rather than arguing for or against this or that definition of martial arts, Wetzler deconstructs and reveals the limits of a range of conventional and popular categories that circulate within martial arts discourses and points to the essential impossibility of establishing fixed referential categories in these waters (Wetzler 2015, 28). He proposes instead that martial arts studies analyses should be orientated by looking for and at

${ }^{10}$ My own contribution was entitled 'Asking the Question: Is Martial Arts Studies an Academic Field?' (Bowman 2015b) In this chapter, I stopped short of explicitly addressing the question of which particular theories or approaches the field might involve (even though my preferences are surely readily inferable). 
the 'dimensions of meaning' attendant to any given construct of martial arts. To this end, he proposes five plausible but always provisional dimensions of meaning: Preparation for violent conflict, play and competitive sports, performance, transcendent goals, and health care. After making a case for these dimensions and inviting others to expand or refine his conceptualisation of them, Wetzler turns to the matter of how to conceive of, frame, and conceptually manage (in order to analyse and discuss) matters of martial arts studies without falling into what Derrida would call 'metaphysical traps', what cultural theorists would call 'essentialisms', and what Wetzler himself calls pitfalls of 'lexical illusion'.

The way to avoid making conceptual mistakes, Wetzler argues, is to find an adequate theory. The one he proposes as valid and viable for martial arts studies is Even-Zohar's polysystem theory. Wetzler quotes the following important passage from Even-Zohar:

Systems are not equal, but hierarchized within the polysystem. It is the permanent struggle between the various strata ... which constitutes the (dynamic) synchronic state of the system. It is the victory of one stratum over another which constitutes the change on the diachronic axis. In this centrifugal vs. centripetal motion, phenomena are driven from the centre to the periphery while, conversely, phenomena may push their way into the centre and occupy it. However, with a polysystem one must not think in terms of one centre and one periphery, since several such positions are hypothesized. A move may take place, for instance, whereby a certain item (element, function) is transferred from the periphery of one system to the periphery of an adjacent system within the same polysystem, and then may or may not move on to the centre of the latter. (Even-Zohar 1990, 13-14, quoted in Wetzler 2015, 28-29)

Wetzler goes on to explain how this theoretical paradigm might be used in martial arts studies:

Transferred to the development of the Asian martial arts in Western culture within recent decades, this means: The total realm of the martial arts is the polysystem in question, which can itself be understood as a system within the ultimate polysystem 'culture'. The cultural meaning of the polysystem 'martial arts' is not monolithic, but instead consists of several systems that each have their own relevance within the polysystem. Such systems might be 'use for self-defence' or 'preferred way of combat for the silver screen', while the 'items' that occupy these systems are the individual martial arts styles. (Wetzler 2015, 28)

Furthermore, the theory seems to offer ways to conceptually grasp change within and across systems. Wetzler continues: 
To clarify with an example: Upon its arrival in the West, karate was perceived mostly for the Dimension 1: Preparation for Violent Conflict, and thus at the centre of the system 'self-defence. However, it has been driven to the periphery of 'self-defence' by other styles, especially by wing chun, which was then in turn driven from the centre by krav maga. Regarding the perception of Dimension 2: Play and Competitive Sports, karate was again driven from a centre, this time of the category 'tough combat sport', in this case by kickboxing, which was replaced by Muay Thai, which was replaced by MMA. However, not all is lost for karate. When the style held the centre of the self-defence system, it also had a connotation of being a pastime for bullies and hooligans. While losing the centres of those systems karate was able to gain ground in the systems including 'martial arts for pedagogical purposes' and 'selfperfection by Eastern practices' (both systems obviously representing Dimension 4: Transcendent Goals), whose centres it shares today with other Japanese budo styles, along with yoga, qigong, and various meditation practices in the second case. (Wetzler 2015, 28)

Wetzler's ensuing discussion of the insights that such an approach opens up is extremely suggestive and rewarding - even though it does not broach the matter of how anyone might ever establish what is at the 'centre, 'periphery' or other 'position' of this or that 'system' - all of which will surely always be in question. Nonetheless, it has already generated (or at least enriched) some highly significant work, most notably in the form of Benjamin N. Judkins' recent study of the Star Wars inspired phenomenon of Lightsaber combat (Judkins 2016a).

Using the 'five dimensions of meaning' that Wetzler proposes can be associated with martial arts practices in different configurations at different times and in different places, Judkins easily demonstrates that the perhaps unlikely pastime of Lightsaber combat training can in fact entirely reasonably be classed as a martial art. This is so even though such a conclusion might surprise or dismay certain scholars of martial arts and even if many of Lightsaber combat's own practitioners would not feel entirely comfortable making such a claim.

Judkins' approach to the quite possibly controversial example of Lightsaber combat, informed by Wetzler's intentionally rigorous (looking) framework, has the benefit of challenging quite a few different positions - including, most importantly, any essentialist or 'referentialist' approach that proceeds on the assumption that something is a martial art if it is somehow 'obviously' a martial art. So, such works as these by Wetzler and Judkins - along with the arguably even more radical approach taken in the recent work of Chris Goto-Jones, who argues that certain kinds of computer gaming can become martial arts practices (Goto-Jones 2016) - are all valuable, and not least because they foreground the limitations of any hasty attempt to define martial arts. Moreover, not only do such approaches all problematize the impulse to rush to definitions, they also 
do so without sidestepping or avoiding the issue of how to specify and handle martial arts as an object of academic attention.

For my purposes, a key value in this work is the demonstration of the primacy and productivity of theory before definition. Such frameworks clearly exceed the frames and orientations of hoplology, for instance, which is mired in inessential preconceptions and doxa. As such, it is in full support of Wetzler's efforts and in broad agreement with the orientations of such scholars that my present contribution to this debate about definition and theory aspires to be read. This is so even though my own contribution does involve criticisms of Wetzler's proposed theoretical paradigm for martial arts studies. But these are less like fundamental disagreements and more like questions for further consideration. Importantly, any criticisms I have will neither be 'anti-theory' nor 'pro-definition'. Rather, in what follows, I seek less to disagree with Wetzler and more to point out some potential pitfalls and problems attendant to any avoidance of theory or insistence on definition in martial arts studies.

\section{Defining Problems: Relationality before Definition}

A well-known part of the problem that arises when trying to define the objects or foci of martial arts studies is semiotic openness, slippage, instability and the incessant ongoing changes that take place across cultures, communities, societies, technologies and practices. Wetzler tackles this by proposing a framework for structuring academic enquiry and proffering a set of theoretical terms for grasping what he represents as 'systemic' but what I would prefer to call discursive change. I prefer to approach the world in terms of the language of texts and discourses rather than elements, functions, systems/polysystems, and so on, for ontological reasons that boil down to the primacy (proposed by poststructuralist theory) of relationality rather than notions of 'system' or even 'systematicity'. As Derrida writes of 'system':

If by 'system' is meant - and this is the minimal sense of the word - a sort of consequence, coherence and insistence - a certain gathering together there is an injunction to the system that I have never renounced, and never wished to. This can be seen in the recurrence of motifs and references from one text to another in my work, despite the differing occasions and pretexts ... 'System', however, in a philosophical sense that is more rigorous and perhaps more modern, can also be taken to mean a totalization in the configuration, a continuity of all statements, a form of coherence (not coherence itself), involving the syllogicity of logic, a certain syn which is no longer simply that of gathering in general, but rather of the assemblage of ontological propositions. In that case deconstruction, without being anti-systematic, is on the contrary, and nevertheless, not only a search for, but itself a consequence of, the fact 
that the system is impossible; it often consists, regularly or recurrently, in making appear - in each alleged system, in each self-interpretation of and by a system - a force of dislocation, a limit in the totalization, a limit in the movement of syllogistic synthesis. Deconstruction is not a method for discovering that which resists the system; it consists, rather, in remarking, in the reading and interpretation of texts, that what has made it possible for philosophers to effect a system is nothing other than a certain dysfunction or 'disadjustment', a certain incapacity to close the system. Wherever I have followed this investigative approach, it has been a question of showing that the system does not work, and that this dysfunction not only interrupts the system but itself accounts for the desire for system, which draws its élan from this very disadjoinment, or disjunction. On each occasion, the disjunction has a privileged site in that which one calls a philosophical corpus. Basically, deconstruction as I see it is an attempt to train the beam of analysis onto this disjointing link. (Derrida 2003, 3-4)

Systems fail to be systematic; system is impossible. This also accounts for the desire for it, and the possibility of the deconstruction of it. Adding 'poly' to the word 'system' does not solve, resolve or dissolve the matter. Pluralizing merely defers acknowledging the fact that there may be no system other than in the 'lexical illusion' of the eye that wishes to perceive/believe that there is systematic organisation and some kind of systematic process at work, even if we can only ever 'discover' (invent) it afterwards.

As an alternative to what Derrida would call 'metaphysical' thinking about systems, the poststructuralist notions of text and discourse provide alternative concepts, metaphors, vocabularies and paradigms (Laclau and Mouffe 1985; Mowitt 1992; Bowman 2007). Key here are the notions of relation or relationality, on the one hand, and force, on the other. It seems worthwhile to discuss these notions further, as they are important dimensions, but they are currently undeveloped, or at best underdeveloped, in Wetzler's proposed framework for analysis in martial arts studies.

To start with the matter of relation first: Can an identity ever be said to be anything other than relational? As Ernesto Laclau and Chantal Mouffe argued in the 1980s, 'identities are purely relational' so 'there is no identity which can be fully constituted' (Laclau and Mouffe 1985, 111; Bowman 2007, 18-19). Already this kind of perspective, with origins in Saussurean linguistics and semiotics, problematizes the notion of 'elements' within a 'system' and replaces the notion of 'entities with identities' with a much more fluid sense of their ongoing incompletion and irreducible contextuality.

Almost two decades after his influential 1985 monograph with Chantal Mouffe, in a dispute with Slavoj Žižek about politics and society, Laclau was still making the same arguments. In response to Žižek's now infamous (and what Laclau always regarded as ill-thought-through) adoption of a kind of crude 
Marxist and quick Leninist position on the question of how to make radical political change in the world, Laclau argued that:

We gain very little, once identities are conceived as complexly articulated collective wills, by referring to them through simple designations such as classes, ethnic groups and so on, which are at best names for transient points of stabilization. The really important task is to understand the logics of their constitution and dissolution, as well as the formal determinations of the spaces in which they interrelate. (Butler, Laclau, and Žižek 2000, 53)

Laclau pitched his argument about how to approach political entities, identities and processes in terms of the vocabulary and concerns of a poststructuralist and post-Marxist political theory, whose essential proposition runs like this: Because everything - and by 'everything' what is meant is everything - can be seen to be contingent and hence conventional, everything is therefore to be regarded as irreducibly political (Arditi and Valentine 1999; Marchart 2007).

There is much to be said about this argument (Bowman 2007, 2008). I return to it here not just as a rejoinder to Wetzler's metaphorical invocation of the putatively non-metaphorical notion of 'system' but also because I believe it is vital (and vitalising) to try, as Laclau urges us, 'to understand the logics of [the] constitution and dissolution [of entities and identities], as well as the formal [or informal] determinations of the spaces in which they interrelate'.

This is important not least because, if 'identities' can also be understood as 'complexly articulated collective wills', then to understand either 'wills' or 'identities' as arising 'systematically' could have a problematic impact on the way we understand such important matters as (for example) political struggle. Stated bluntly, to rely on polysystem theory might cause us to follow a line of thinking in which political struggles and political identities come to be conceived as somehow merely being the systematic unfolding of some kind of predetermined plan.

This is why the notion of force is also key. Entities and identities are not just matters of signification, or of systems, but also of force. Force is the other side of signification, a key part of the process of establishing meaning (Protevi 2001). This is why Laclau believes we should not be content with the moment of referring to entities and identities 'through simple designations such as classes, ethnic groups and so on': Because such terms 'are at best names for transient points of stabilization'. In other words, signification should not be studied in isolation from considerations of force. ${ }^{11}$

${ }^{11}$ Laclau's use of the word 'stabilization' here is significant. It seems to owe something to the fact that Derrida once emphasised the importance of the ideas of stabilization and destabilization in a published conversation with Laclau in the 1990s (Mouffe 1996). In his response to Laclau and others, Derrida said: 'All that a deconstructive point of view tries to show, is that since convention, institutions and consensus are stabilizations (sometimes stabilizations of great duration, sometimes micro-stabilizations), this means that they are stabilizations of something 
So, Laclau's broadly deconstructive perspective challenges us to think about the making or establishment of any identity in a way that exceeds the lexical illusion of systematicity and emphasizes instead the complexity of contingent processes of articulation (Laclau 1994). This differentiated perspective - which replaces ideas of structures and systems with those of iteration, reiteration, dissemination, dislocation, and so on - forms the main part of my critique of the use of polysystem theory in martial arts studies, or at least my critique of Wetzler's advocation of it. However, to reiterate, making such a critique is not my primary aim here. Wetzler is a sparring partner, not an opponent. Rather, the matters that I ultimately want to challenge are somewhat different.

\section{Changing Discourses}

Specifically, I want to point out that Laclau's approach to discourse analysis involves rather different investments than thinking about the academic definition of any activity, entity or identity. Indeed, although Laclau's argument here includes the injunction that academics be rigorous and forensic in their conceptual grasp of their key terms, it is not limited to this injunction. Moreover, the position Laclau advocates does not merely involve the endless or supposedly 'useless' problematizing of terms (something deconstruction was once regularly accused of), whether to try to reconfigure and refine the definitions and distinctions that academics use in their work or those that practitioners use in their practice, or to show them to be impossible.

Rather, for Laclau - and indeed for the overwhelming majority of works of cultural theory developed through and since the 1980s - the fundamental point to be taken on board is not that we should work out how best to define something; it is rather that we must face up to the fact that 'things' are neither simply nor necessarily 'things', that all identities are at root contingent discursive achievements, or establishments, or - to use Laclau's words, 'transient points of stabilization.' ${ }^{12}$

essentially unstable and chaotic. Thus it becomes necessary to stabilize precisely because stability is not natural; it is because there is instability that stabilization becomes necessary; it is because there is chaos that there is a need for stability. Now, this chaos and instability, which is fundamental, founding and irreducible, is at once naturally the worst against which we struggle with laws, rules, conventions, politics and provisional hegemony, but at the same time it is a chance, a chance to change, to destabilize. If there were continual stability, there would be no need for politics, and it is to the extent that stability is not natural, essential or substantial, that politics exists and ethics is possible. Chaos is at once a risk and a chance, and it is here that the possible and the impossible cross each other' (Derrida 1996, 84).

12 Accordingly, given that 'martial arts studies' takes its very name and focus ('martial arts') from what Wetzler deems to be the dubious and problematic realm of 'object language', there can therefore be no 'metalanguage' that is not contaminated by this fact. As Laclau and Mouffe argued in the 1980s, because there is never anything like a fixed centre, stable system or simple outside, there can be no metalanguage (1985). 
Moreover, our shared use of a term like 'martial arts' or 'system' stabilizes our discourse. But it can also impose and project a fixed view - our present view - of all sorts of dimensions of culture and society, both backwards in time and outwards across different linguistic, geographical, cultural, religious and social contexts. So, the establishment of a shared and stable term has its benefits (predication and communication being among them). But it inevitably also comes at a cost - which we might render in a number of ways, including projection, simplification, hypostatisation, generalisation, transformation, or even cultural, conceptual or linguistic imperialism.

Wetzler calls this 'lexical illusion', as in: We say 'martial arts' in English here today, but did or do they say or mean anything like it there (elsewhere) or then (elsewhen), without difference or remainder? Or are we misrecognising the things 'out there' (and 'then') that we talk about in our terms, here and now? As an example, consider how frequently it is currently said that 'mindfulness meditation' has been practiced within Eastern movement traditions and martial arts for millennia. (Before we heard this claim being made about mindfulness, we heard the same claim being made about qigong [Palmer 2007]. And before that, it was said about yoga [Spatz 2015]. And so on.) Such propositions are all based on acts of fantasy and projection, back into a fantasized notion of 'long, long ago' (Fabian 1983).

Such acts of projection are clearly faulty. They also have any number of potential ideological dimensions and material and discursive effects. Consider a second example. On a tour I was given during a visit to the new Mecca of Taekwondo in South Korea, the Taekwondowon, our guide pointed to a picture of an old statue and said, 'Look, this is a statue of someone doing taekwondo. That posture comes from taekwondo'. The facts that (a) taekwondo was only invented in the 1950s (Gillis 2008; Moenig 2015) and (b) its patterns, or kata, were only subsequently changed from the Japanese martial arts from which it was derived would seem to problematize the idea that an ancient statue could possibly depict a taekwondo posture. The possibility that the taekwondo posture might have been invented deliberately to depict the ancient statue in order to strengthen the ideological claim that taekwondo is ancient was not really encouraged or entertained at all. ${ }^{13}$

Entities and identities are discursive achievements, produced through efforts and institutions, arguments, articulations, demonstrations, and indeed processes and acts of institution (where 'institution' is to be read as both noun and verb). What something 'is' emerges through forceful - often enforced processes of narration and representation. 'Mindfulness' is an entirely modern construct. 'Taekwondo' is no older than the 1950s. The resignification of such

\footnotetext{
${ }^{13}$ After my visit, I blogged about this here: https://goo.gl/FXVF6T. I also went on to discuss it in 'Making Martial Arts History Matter' (Bowman 2016) and in Mythologies of Martial Arts (Bowman 2017b).
} 
institutions as ancient is an effect of the contingent but motivated modes and manners of their discursive articulation and emergence.

\section{Optimistic Relations}

Theoretically, I have revisited some broadly poststructuralist points (all too) briefly here because I believe that remembering and taking into consideration these lessons in our various ongoing research projects into martial arts - and the international development of the field of martial arts studies - will allow us to move on, and specifically to move on from a certain kind of fixation on definition. (Neither Wetzler nor Judkins suffers from this fixation, however, and my comments about the problems with definition, though inspired in part by engagements with their work, are not directed towards either of these scholars.)

I am drawing attention back to poststructuralist theory because, rather than orientating and habituating us into an academic life of taxonomical labours centred on defining and demarcating, such approaches proceed from the proposition that identities are always irreducibly relational and incomplete, and hence contingent, open and ongoing. Identities are constituted by and within discourses, and they always emerge as points in clusters of moving constellations of related, contiguous, cognate, differentiated, associated, contrasting and oppositional terms, in all kinds of possible relations - linguistic, semiotic, lived, institutional, academic, legislative, and so on.

One point to be emphasised again is the role not just of lexical illusion but also of force within the construction of entities and identities. Whether using what Wetzler terms object language or what Derrida terms metalanguage, we always think through and with inherited terms, and hence conceptual differentials and differentiations - inheritances that we are more or less forced to work with and, to some degree, within (Derrida 1976). ${ }^{14}$

${ }^{14}$ Nonetheless, as Saussure taught us, when we are thinking about our linguistically instituted categories, first and foremost we must remember that there are only 'differences without positive terms'. Moreover, as Derrida went on to demonstrate, there are no easily specifiable or simply stable referents 'behind' these differences. The flipside of signification is force (Protevi 2001). There is no stability in signification without force. Furthermore, as Gayatri Spivak added, the institution of any difference in the production of an identity in discourse, the drawing of any demarcation that distinguishes and hierarchizes entities and identities, is essentially and irreducibly a political act, with more or less overtly political consequences (Spivak 1990, 1993). (Such poststructuralists sometimes even formulate dimensions of this in terms of violence [Bowman 2010a]. This means that, if we were to follow this logic through to one of its conclusions, it would become possible to argue that more or less any identity is in some sense 'martial' (it has either been fought for or fought against), as well as stabilized but conflictual.) Within martial arts studies, quite what these acts and their consequences may be remains to be seen. But hopefully such reflections as this may cause some hesitation, and possibly reorientation, before the battles continue over this or that 'correct' definition. I return to this point at the end of this chapter. 
Now, although I am critical of the scramble for definitions, nonetheless, it strikes me that the growing prominence of the matter of definition does attest to a lot that is promising in the current stage of development of martial arts studies. It is evidently a reflection of the drive to found and ground and legitimate and build the field rigorously, and according to proper academic protocols. To this extent, despite the scientistic features of some forays into this new terrain, our current moment is of great significance. So, we may be optimistic. However, in the current rush to try to define and establish 'things', there is always the risk that we labour under misapprehensions. My concern is that some of the misapprehensions we see arising today may come to constitute an obstacle or impediment in the development of the field tomorrow, pushing it towards becoming something dominated by what Žižek once termed 'naïve empiricism' or 'naïve cognitivism' (Žižek 2001a). Decades before Žižek, Derrida too had worried about something similar, which he called 'incompetent' and even 'irresponsible' empiricism (Derrida 2001).

What such thinkers mean in making claims like 'empiricism is naïve, incompetent, or even irresponsible' - is that there is a kind of untenable idealism and simplicity at the heart of approaches that begin from the premise that to make sense of the world we should simply look around us, focus on things, classify them and count them, and that, through a process of testing and disputing around categories, we might eventually get at the truth of reality and get it right. Their more or less opposite opinion is that, on the contrary, what we all always need is an explicit theory. I say explicit theory, and not just 'theory', because, arguably, everyone always has a theory, even if they don't consciously know what it is. By 'explicit theory' I am referring to anything from an overarching theory of ontology to an actively thought-through image or sense (to use Laclau's terms again) of how discourses and identities are constituted and the logics of their processes of establishment, stabilization, interaction, transformation, and dissolution. ${ }^{15}$

It is in this sense that I am arguing for more theory, an injection of theory, and the permeation of theory, before definition. But I am not proposing a return to the intellectual battles of the 1980s and 1990s, in which the introduction of Continental Philosophy into the humanities led to a state of trench warfare between those who 'did theory' and those who 'did empirical work' (Hall 2002). Furthermore, although I am arguing explicitly 'for theory', I want to be clear that I am certainly not therefore arguing 'against empirical work', or 'history', or 'reality', or anything like that.

Rather, I want to insist that it will be vital and vitalising for work in martial arts studies to embrace certain aspects of cultural theory, especially when - as in the current moment - people seem to feel an apparent 'need' to do something

${ }^{15}$ I use the word 'sense' here because I think that we can only ever get an image, sense or feeling for ontology anyway. I hesitate to say 'structure of feeling', though, for, as Derrida himself made clear, the very idea, term, notion or (possible) concept of 'structure' is rarely ever much more than a metaphor anyway. 
properly academic, a need that so many people seem to believe is to be interpreted as defining our object. For, faced with the (apparent) challenge of 'needing' to define, as we have already seen, with even the tiniest bit of theory, we are able to pause to reflect on the fact that before definition there is relation. Words and meanings and practices and values travel and twist and turn and change and move in relation to larger and other forces and processes. These may or may not be systemic, systematic (Wetzler, Even-Zohar), conjunctural (Hall), discursive processes of articulation (Laclau), or 'dislocated', 'out of joint' or even 'hauntological' (Derrida 1994), and so on.

All such theories would concur that martial arts will always be relationally determined. Laclau and Mouffe theorised this in terms of 'discourse' and 'articulation' (Laclau and Mouffe 1985). Hall insisted on the need to establish a sense of what he called the 'conjuncture'; according to him, any analysis requires what he called 'conjunctural analysis' - that is, an analysis informed by an acute awareness of the historical moment and context as well as the forces and relations that produced it. Without this, we cannot really know or understand anything about any entity or identity, whether martial arts, class, ethnicity, or any other kind of identity or entity in process.

Of course, there may be many ways to characterise and analyse a conjuncture. As deconstruction sought to teach us, no context is ever fully closed (Derrida 1988). We might never know for sure that we know for sure everything salient about a context or a conjuncture. Maybe we can't really know for sure that we know anything at all for sure. Yet, what we can do is attempt to assess a context in terms of forces and relations, relative weights and gravities, and the ways in which forces and fields constitute, colour and condition entities, identities and practices. This may not be too far from Wetzler's proposed use of Even-Zohar's polysystem theory - or it may be a world away.

\section{Alternative Discourses}

In this chapter, I have so far proposed the necessity of theory for martial arts studies and entered into a critique of one proposed branch of theory. I have done so because part of what needs to be theorised is the orientation of the discipline's discourse, and I would prefer to steer that discourse as far away from anything approaching scientism as possible. My chief criticism of the tropes of 'systems' would be that this approach risks pointing the discourse of martial arts studies back towards a scientistic orientation.

Given this criticism, an obvious question is what, therefore, my proposed alternative approach would be. My answer relates to my ongoing arguments from poststructuralism about the need for attention not just to signification ('dimensions of meaning') but also to force, as in the forms of different relations to and entanglements within different kinds of social, cultural, economic and other forms of power. 
In Britain, Raymond Williams long ago proposed that it is possible to formulate and look at entities, practices and identities and to assess them in terms of whether they are dominant, residual or emergent, and to ask whether they may be acting in ways that are either in line with a dominant or hegemonic ideology, or whether they may be alternative or even oppositional to such an ideology (Williams 1977). This may seem like quite an old and crude paradigm. This kind of approach has certainly been significantly refined and developed over the decades (Laclau and Mouffe 1985; Laclau 1994; Butler, Laclau, and Žižek 2000). But I draw attention to this seminal paradigm here because, even as simple as it is, it offers a viable and flexible framework through which many different kinds of studies of martial arts and society might be initiated. All of these are happily liberated from the stifling imperative to define and demarcate without any real sense or sensitivity to the complexities of matters of time, place and the interplay of forces that both produce and transform meanings, practices and contexts.

To illustrate the value of this framework, we might quickly consider one final example: The deliciously marginal or problematic case of taijiquan. Using Williams' approach, we will be able to reconfigure discourse and debate about taijiquan from a sclerotic fixation on the question of whether it can even be 'defined' as a martial art or a combat sport, or self-defence, or a form of what we now insist on calling 'mindfulness meditation', etc., and to an understanding of what 'taijiquan' has been and has done and might be and might do in a given conjuncture. ${ }^{16}$

As Douglas Wile has argued, taijiquan emerged in a discursive foment in which China was threatened ideologically, economically and politically (Wile 1996). Its $19^{\text {th }}$ Century proponents elaborated its philosophy along obscurely yet immanently nationalist lines, so that taijiquan came to stand in stark opposition to any and all things Western or European (see also Lorge 2016). In this process, residual Taoist ideas and principles were mixed into a growing alternative worldview that was oppositional to everything supposedly non-Chinese. This is also precisely why Maoism tolerated taijiquan, of course, and why it 'survived' the Cultural Revolution: It amounted in its elaboration to a collective, combined, non-Western, non-competitive, non-individualistic calisthenics avowedly rooted in a non-religious worldview. But this was 'survival' via a formalisation that amounted therefore to a mutation on a genetic level. So, in a sense, post-Mao, the term taijiquan essentially had a transformed meaning referring to a transformed practice (Frank 2006).

In its journey to the West, as we know, taijiquan was ostensibly deracinated from any nationalistic inflection or valence, and became articulated to

${ }^{16}$ Note again the way that we now 'see' 'mindfulness' everywhere, from meditation in modern America to martial arts in ancient China, even though even a few years ago we wouldn't have seen anything as mindfulness, anywhere, because no one, other than a few specialists, was using the term. 
(connected with) a range of open-ended discursive configurations or conjunctures, from the counterculture to new age ideology and onwards into therapeutic and even medical culture (Frank 2006). In all this, it becomes differently articulated or constructed at different times and places, often existing with utterly contradictory and heterogeneous (non-systemic, non-systematic) partial, immanent or potential meanings at the same time. Furthermore, any of those involved in taijiquan in any of its different times and places will believe themselves to be either or both learning a martial art, either or both for sport or for self-defence, and/or involved in healthful calisthenics, and/or preserving or changing a culture, and/or involved in a religious or mystical practice. And so on.

We can multiply our examples to look at the ways in which certain words and moves have drifted and disseminated and flipped and mutated all over the place, around the world, through time and space, and examine the processes of their emergence and development within each new context, the ways they become mixed up and mixed in with existing concerns and outlooks, and reciprocally modify and move existing situations. This may or may not be systemic or systematic.

I have mainly referred to the theoretical models of people like Laclau, Derrida, Hall and Williams here. And I have done so mainly because I believe that there is - to a greater or lesser extent - a kind of theoretical ontology that connects their outlooks, despite their many other differences. This outlook is essentially poststructuralist or postfoundationalist (Sedgwick 2003). ${ }^{17}$ And as much as many people may still have a distaste for so-called 'high theory', I maintain that martial arts studies will only benefit from a sustained engagement with what there is to be learned from high theory - as much as there is to be learned from engaging with the most intimate ethnography, the most detailed historiography, the most multi-layered sociology, and so on.

Some of the first lessons relevant to us here would relate to an awareness of the slippage and vicissitudes of signification that require us to pay very close attention to the shifting and drifting apparent referents of our focus, their different meanings in different times and places, the genetic mutations and quantum leaps that occur in 'cultural translation' from one time to another, one place to another, one language to another, even one utterance or instance to the next, and the rather frustrating fact that, despite our eternal desire to see unity and simplicity, cultures and practices are always 'in bits', always in process, incomplete, disputed and contested. There is no unity to the lexical illusion that guides us, whether it be martial arts, combat sports, self-defence, culture or society - apart from that which seems to be conferred by the use of such terms themselves.

${ }^{17}$ Interestingly, Sedgwick (2003) also sees an affinity between poststructuralist and Buddhist ontologies, and she ponders whether she is drawn to the former because of her interest in the latter or to the latter because of her agreement with the former. 
Discussing such entities often has much in common with discussing unicorns, fairies, justice, Father Christmas, or how many angels might fit on the head of a pin or through the eye of a needle. Discussing such things can create a 'reality effect' that can lead people to believe these are actually existing real and unitary things (Bowman 2012). All meanings, all practices, are stabilizations. The questions to be asked then surely include explorations of why certain stabilizations take place at certain times in certain ways, why some people often become so fixated on fixation or stuck on stabilization, and what it is that both stabilization and destabilization are 'doing' in any given context at any given time.

\section{The Stabilization of Martial Arts}

Martial arts as a cluster of familiar ideas, motifs, images, and as a category has certainly achieved stabilization in contemporary discourses, even if it lacks both precision and a stable referent. Nonetheless, it is a relatively stable term perhaps no more or less stable or precise than any other familiar term, such as 'society', for instance. That is to say, despite its familiarity, the term 'society' could have a number of different conceptualisations and configurations, and it could mean different things within different configurations.

This 'semiotic openness' around even the most familiar terms is interesting. Even more interesting is the fact that the most widespread scholarly response to semiotic openness and instability is not to embrace it and explore it, but rather to try to close it down and eradicate it, by such strategies as imposing definitions and insisting upon strictly demarcated meanings. Such responses seek to eradicate or banish predicative instability, in order to try to be clear. Accordingly, such an impulse is understandable. Nonetheless, one problem with it is that academic definitions and strict meanings often give short shrift to the ways that terms actually circulate and function in the discourses of the everyday lives of the people who use the terms out there in the world. Consequently, this form of stabilization may come to be problematic, especially if we are indeed interested in the things as they exist 'out there' rather than as they might be made to seem to exist within a scholarly discourse.

Of course, the meanings and definitions generated in scholarly discourse often come to inform, enrich and even transform the meanings of terms as they circulate in wider cultural discourse. But self-reflexive scholarship ought to interrogate this relation rather than just assume everything is straightforward. It may turn out to be valuable to work out where a term and its meaning came from - was it inherited from 'folk' or 'popular' discourse, or was it generated in a laboratory, so to speak? And what are the consequences of either inheritance? There is always drift and condensation and displacement going on. We think through and with inherited terms, and hence conceptual differentials and differentiations, that we are more or less compelled to work with and within (Derrida 1976). But, as Saussure taught us, when we are thinking about 
our linguistically instituted categories, first and foremost we must remember that there are only 'differences without positive terms'. Moreover, as Derrida went on to point out, there are no easily specifiable or simply stable referents 'behind' these differences. The flipside of signification is force (Protevi 2001). There is no stability in signification without force. Furthermore, as Gayatri Spivak added, the institution of any difference in the production of an identity in discourse, the drawing of any demarcation that distinguishes and hierarchizes entities and identities, is essentially and irreducibly a political act, with more or less overtly political consequences (Spivak 1990, 1993). But what is being stabilized, and in what ways? This question supplements the next chapter. 
